\title{
STATUS AND PERSPECTIVES OF THE MINI-MEGATORTORA WIDE-FIELD MONITORING SYSTEM WITH HIGH TEMPORAL RESOLUTION
}

\author{
Sergey Karpov ${ }^{a, *}$, Grigory Beskin ${ }^{a}$, Sergey Bondar ${ }^{b}$, Alexey Perkov ${ }^{b}$, \\ Evgeny IVANOV ${ }^{b}$, AdRIANO GUARNIERI $^{c}$, CORRAdo BARTOlini $^{c}$, \\ Giuseppe Greco $^{d}$, Andy Shearer ${ }^{e}$, Vyacheslav SasyuK $^{f}$ \\ a Special Astrophysical Observatory of the Russian Academy of Sciences \\ ${ }^{b}$ Institute for Precise Instrumentation, Russia \\ ${ }^{c}$ Bologna University, Italy \\ $d$ Astronomical Observatory of Bologna, INAF, Italy

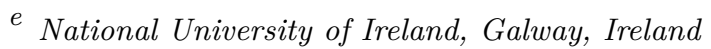 \\ $f$ Kazan Federal University, Kazan, Russia \\ * corresponding author: karpov@sao.ru
}

\begin{abstract}
Here we briefly summarize our long-term experience of constructing and operating wide-field monitoring cameras with sub-second temporal resolution to look for optical components of GRBs, fast-moving satellites and meteors. The general hardware requirements for these systems are discussed, along with algorithms for real-time detection and classification of various kinds of short optical transients. We also give a status report on the next generation, the MegaTORTORA multi-objective and transforming monitoring system, whose 6-channel (Mini-MegaTORTORA-Spain) and 9-channel prototypes (Mini-MegaTORTORA-Kazan) we have been building at SAO RAS. This system combines a wide field of view with subsecond temporal resolution in monitoring regime, and is able, within fractions of a second, to reconfigure itself to follow-up mode, which has better sensitivity and simultaneously provides multi-color and polarimetric information on detected transients.
\end{abstract}

KEYWORDS: gamma-ray bursts, high time resolution, wide field photometry.

\section{INTRODUCTION}

The systematic study of night sky variability on subsecond time scales remains an important but practically unsolved problem. The need for such a study for the purposes of the search of non-stationary objects with unknown localization has been noted by [10]. Such studies have been performed [24, 25, but due to technical limitations it has only been possible either to achieve high temporal resolution of tens of milliseconds in monitoring of $5^{\prime}-10^{\prime}$ fields, or to use $5-10$ seconds time resolution in wider fields. The wide-field monitoring systems currently in operation, such as WIDGET 27, RAPTOR [11, BOOTES [13 and Pi of the Sky [12, while having good sky coverage and limiting magnitude, lack temporal resolution, which significantly lowers their performance in the study of transient events of subsecond duration.

Optical transients of unknown localization may be very short. For example, the rise times of flashes of some UV Cet-like stars may be as short as $0.2-$ 0.5 seconds [26], $30 \%$ of GRBs are shorter than 2 seconds in duration, and details of their light curves may be seen on time scales shorter than $1 \mathrm{~ms}[20$. Also, of great interest are observations of very fast meteors (faster than $200 \mathrm{~km} / \mathrm{s}$ ) which may be of extraSolar system origin [1. Slower meteors should also be studied with high temporal resolution due to their short duration (typically just a fraction of second) and the necessity to observe them on at least two consecutive frames to reliably recover their angular velocity.

Monitoring of near-Earth space is another task that requires wide-field observations with high temporal resolution. A number of satellites, and also a vast amount of small pieces of space debris, have rapidly evolving trajectories, and are therefore difficult to observe by typical narrow-field satellite tracking methods based on extrapolation of previously known trajectories. High temporal resolution is needed here due to the fast motion of these objects in the sky, up to several degrees per second for low-orbit objects, and the fast decrease in the detection limit when there is significant motion of the source over the exposure time due to spread of its flux across several pixels along the trail.

It has been proposed [9] that large low-quality mosaic mirrors of air Cerenkov telescopes should be used to study the variability of large sky areas on such time scales. However, we have demonstrated in [16, 30] that subsecond temporal resolution can be achieved in a reasonably wide field with small telescopes equipped with fast CCDs, to perform fully automatic searching 


\begin{tabular}{lccc}
\hline Name & $\begin{array}{c}\text { FOV } \\
(\text { degrees })\end{array}$ & $\begin{array}{c}\tau \\
(\text { seconds })\end{array}$ & $\begin{array}{c}\text { Limit } \\
(\text { magnitudes })\end{array}$ \\
\hline WIDGET & $62 \times 62$ & 5 & $10^{\mathrm{m}}$ \\
RAPTOR A/B & $40 \times 40$ & 60 & $12^{\mathrm{m}}$ \\
RAPTOR Q & $180 \times 180$ & 10 & $10^{\mathrm{m}}$ \\
$\pi$ of the Sky & $40 \times 40$ & 10 & $12.5^{\mathrm{m}}$ \\
AROMA-W & $25 \times 35$ & $5-100$ & $10.5^{\mathrm{m}}-13^{\mathrm{m}}$ \\
MASTER-VWF & $20 \times 21$ & 5 & $11.5^{\mathrm{m}}$ \\
MASTER-Net & $30 \times 30$ & 1 & $9^{\mathrm{m}}$ \\
\hline FAVOR & $16 \times 24$ & 0.13 & $10^{\mathrm{m}}-11.5^{\mathrm{m}}$ \\
TORTORA & $24 \times 32$ & 0.13 & $9^{\mathrm{m}}-10.5^{\mathrm{m}}$ \\
\hline MMT & $30 \times 30$ & $0.13-1300$ & $12.5^{\mathrm{m}}-17.7^{\mathrm{m}}$ \\
\hline${ }^{*}$ Field of view is for a 4-objective unit in wide-field mode; \\
there is also 2-objective unit in operation now.
\end{tabular}

TABLE 1. Wide-field monitoring cameras currently in operation, with field of view (FOV) size, temporal resolution $\tau$ and the detection limit claimed by the authors. For FAVOR, TORTORA and Mini-MegaTORTORA the limits correspond to $3 \sigma$ detection on a single frame, and may differ from their real-time operational values due to non-ergodic pixel statistics when using the image intensifier.

and classification of fast optical transients. In addition, a two-telescope scheme [3, 15] has been proposed which is able to study these transients within a very short time after detection. In accordance with these ideas, we created the prototype of the FAVOR fast wide-field camera [16] and the TORTORA camera as part of the TORTOREM two-telescope complex [21, and we have operated them over a period of several years.

The recent discovery of the brightest ever GRB, GRB080319B (Naked-Eye Burst [23), by several widefield monitoring systems — TORTORA [17], RAPTOR [28] and Pi of the Sky [14 — and the subsequent discovery of its fast optical variability [7] on time scales from several seconds down to a sub-second time scale 8 have demonstrated that the ideas behind our efforts in fast temporal resolution wide-field monitoring are correct.

\section{General REQUiREMEnTS FOR WIDE-FIELD MONITORING}

Typical follow-up observations performed for a detailed study of newly discovered transients require no more than a good robotic telescope with fast repointing. However, instruments of this kind will inevitably only begin to capture data after the event has been in progress for a few seconds or tens of seconds. To obtain information from the start of the event, which is essential for understanding the nature and properties of transients, one needs to observe the position of the transient before it appears. As transients occur in unpredictable places, systematic monitoring of large sky regions is therefore an important task.

For monitoring of this kind, one needs to select the optimal set of mutually exclusive parameters - the angular size of the field of view, the limiting magnitude and the temporal resolution. Indeed, the area of the sky $\Omega$, covered by an objective with diameter $D$ and focal length $F$, equipped with an $N \times N$ pixel CCD with pixel size of $l$ and exposure time of $\tau$ seconds, is

$$
\Omega \propto \frac{N^{2} l^{2}}{F^{2}}
$$

while the faintest detectable object flux, for sky background noise dominating over the CCD read-out noise, is

$$
\text { Flux }_{\min } \propto\left(\frac{D}{F}\right) D^{-2} l \tau^{-\frac{1}{2}}
$$

For the case of CCD read-out noise $\sigma$ domination, the limit is

$$
\text { Flux }_{\min } \propto \frac{\sigma}{D^{2} \tau}
$$

The number of detectable events, uniformly distributed in Euclidean space, is

$$
\text { Number } \propto \Omega \cdot \text { Flux }^{-\frac{3}{2}}=D\left(\frac{D}{F}\right)^{\frac{1}{2}} \tau^{\frac{3}{4}} N^{2} l^{\frac{1}{2}}
$$

when the duration of the event $T$ (or, more accurately, the duration of its peak, where the flux may be treated as a constant) is longer than the exposure, and

$$
\begin{aligned}
\text { Number } \propto \Omega \cdot \text { Flux }^{-\frac{3}{2}} & \left(\frac{\tau}{T}\right)^{-\frac{3}{2}} \\
& =D\left(\frac{D}{F}\right)^{\frac{1}{2}} T^{\frac{3}{2}} \tau^{-\frac{3}{4}} N^{2} l^{\frac{1}{2}}
\end{aligned}
$$

when it is shorter - as Flux min decreases, one can detect a larger number of events in a greater volume. Thus, high temporal resolution is essential in detecting and analysing short optical transients. However, it requires the application of fast CCD matrices. Fast CCD matrices usually have large read-out noise, which limits the detection of faint objects. 


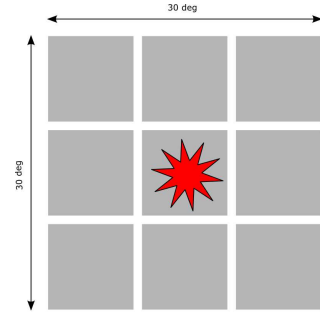

Wide-Field Monitoring

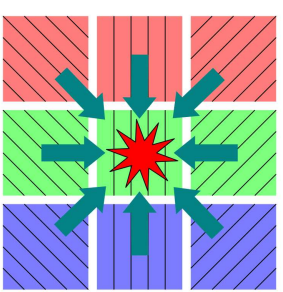

Filters Installation

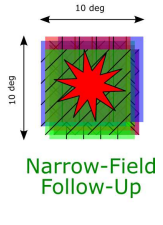

llow-Up
Figure 1. Different modes of operation of the MegaTORTORA system. Left: wide-field monitoring mode in a single color band (or in white light). Center: insertion of color and polarization filters as the first step of the follow-up routine upon detection of a transient. Right: repointing of all unit objectives towards the transient. In the latter mode of operation the system collects three-color transient photometry for three polarization plane orientations simultaneously. The mode transition is expected to be less than 0.3 second.

Most of the general-purpose wide-field monitoring systems currently in operation, listed in Table 1 have chosen a large of field of view while sacrificing temporal resolution to achieve a decent detection limit. By contrast, our cameras, starting with the FAVOR prototype [16, 30, chose high temporal resolution as the key parameter.

\section{MegatoRTORA - MULTI-OBJECTIVE TRANSFORMING INSTRUMENT}

It has been shown above that the parameters defining field of view size, detection limit and temporal resolution are mutually exclusive, and are limited by the difficulties of constructing and using objectives with large relative apertures $(D / F \sim 1$ or greater $)$.

The only possible way to further improve the parameters simultaneously is to design a multi-objective monitoring system, where the detection limit is improved by decreasing the angular pixel size [6] and the field of view is improved by pointing several identical channels towards different regions of the sky. To operate in a sky background dominated regime, the CCD read-out noise may be suppressed by a high quantum efficiency image intensifier, or by using low-noise EM-CCD or sCMOS as a detector.

Multi-objective design also gives freedom in the regimes of operation, as the fields of view of the channels may be either separated or combined, either with the same photometric (or even polarimetric) filter or with a combination of different filters.

The MegaTORTORA project [5] is being developed along these lines. It utilizes a modular design and consists of a set of basic units, 9 objectives each, installed on separate mounts. Each objective in a unit is placed inside the gimbal suspension with remotely-controlled micro-motors, and may therefore be oriented independently from the others. In addition, each objective possesses a set of color and polarization filters that can be installed before the objective on the fly. This enables modes of observation to be changed on the fly, from routine wide-field monitoring in the color band, which provides the best signal-to-noise ratio (or in white light, with no filters installed), to the narrowfield follow-up regime, when all objectives are pointed towards the same point, i.e., a newly-discovered transient, and observe it simultaneously in different colors and for different polarization plane orientations, to acquire all possible kinds of information on the transient (see Figure 1). There can also be simultaneous observation of the transient by all objectives in white light, inorder to obtain better photometric accuracy by co-adding frames.

Each objective is equipped with the fast EM-CCD, which has low readout noise even for high frame rates when internal amplification is in effect. The data from each channel of this system, which is roughly 20 megabytes per second, is collected by a dedicated rackmount $\mathrm{PC}$, which stores it in its hard-drive and also processes the data in real time in a way similar to the current processing pipeline of the FAVOR and TORTORA cameras, which currently operate under a similar data flow rate. The whole system is coordinated by the central server, which acquires the transient data from data-processing PCs and controls the pointing and mode of operation of all objectives in response to them.

\section{Mini-Megatortora as a MEgATORTORA PROTOTYPE}

As a prototype of the MegaTORTORA concept, we designed the Mini-MegaTORTORA, which is basically a model of a $3 \times 3$ unit. The main design choice was to use the celostate in a gimbal suspension for fast repointing of each channel. This decision allows us to significantly loosen the requirements for the structural, dynamical and precision parameters. We are building two variants of Mini-MegaTORTORA with different detectors (an image intensifier with fast CCD for MMT-Spain, and a low-noise sCMOS for MMT-Kazan) and, therefore, with slightly different parameters.

Both variants use the CANON EF85 F/1.2 lens as the main objective and celostate mirrors for fast (faster than $0.3 \mathrm{~s}$ ) repointing in the $\pm 20^{\circ}$ region of the sky. The optical design of the first variant is analogous to the design used in the FAVOR [16] and TORTORA [21] systems, but with a non-scaling image intensifier, see Figure 2, For the second variant, the design is simplified, and lacks the image intensifier and the transmission optics.

The detector of the first variant image intensifier for Spain is based on a fast Sony IX285AL CCD chip with a $6.4 \mu \mathrm{m}$ pixel and $0.13 \mathrm{~s}$ exposure in a continuous acquisition regime, which gives $7.51392 \times 1036$ 


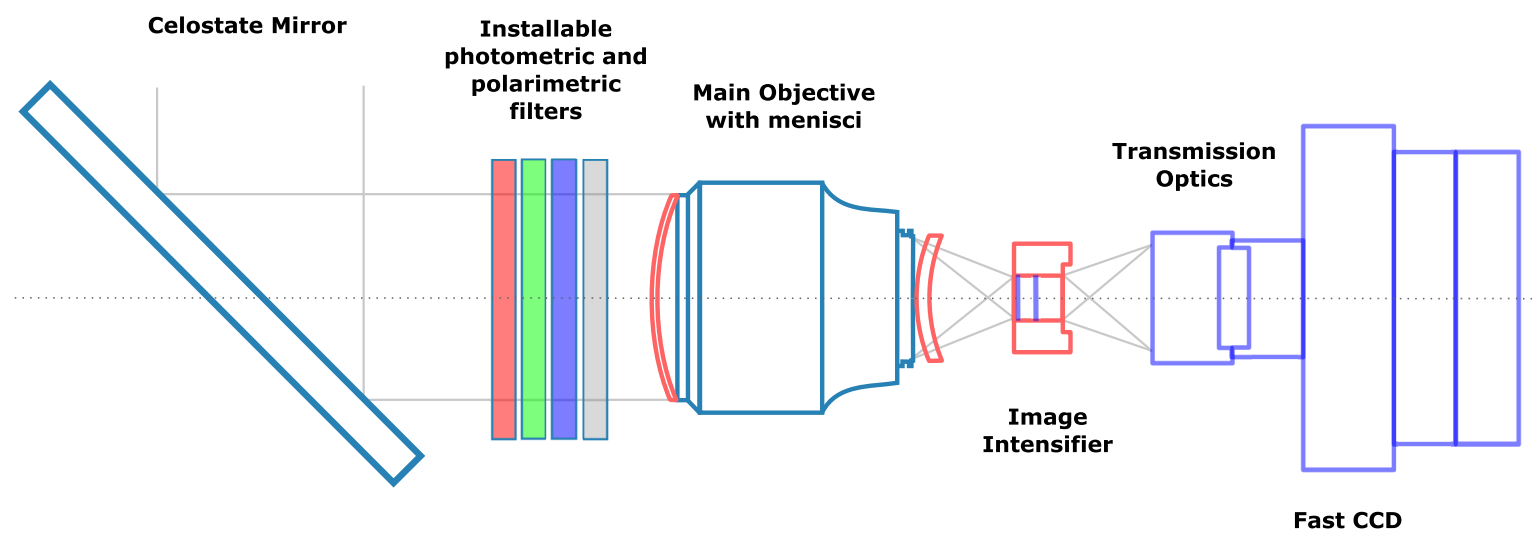

Figure 2. Optical scheme of a single channel for the MMT-Spain variant. The main objective is surrounded by two menisci to compensate the optical distortions of the thick glass on the input of the image intensifier.

frames per second with 12-bit depth. The non-scaling image intensifier has quantum efficiency of about $25 \%$, and the amplified image from its output window is transferred to CCD by transmission optics, which downscales it 1.7 times; the resulting pixel scale is $25^{\prime \prime}$ per pixel, and the total field of view of a channel is about 100 square degrees. The high image intensifier amplification (of $\sim 150$ ) overcomes the CCD read-out noise, but induces its own spatially-correlated and highly non-Poissonian shot-noise due to ions striking the photocathode events. The resulting limiting magnitude in differential imaging mode is about $B \sim 12^{\mathrm{m}}$; it is somewhat worse in the direct imaging regime, due to the spatial correlation of the dominant image intensifier noise. In addition, direct imaging suffers from the non-uniform spatial sensitivity of the image intensifier microchannel plates, which makes it very important to perform proper flatfielding. Each channel is therefore equipped with its own flatfielding module, consisting of a dull surface on the inner part of the lid and dedicated photodiodes.

Due to financial limitations, we are building only 6 channels for this variant, which will simultaneously provide imaging in only two photometric filters (which, of course, may be selected arbitrarily from the three available filters) and three polarimetric filters.

The mechanical scheme of the channel for this variant is shown in Figure 3 .

MMT-Spain will be installed at the El-Arenosillo atmospheric station in Huelva, Spain in fall 2013.

The second variant, MMT-Kazan, is equipped with Andor Neo sCMOS, which has $2560 \times 21606.4 \mu \mathrm{m}$ pixels with 16-bit depth. Due to limitations of the PC processing power, and also limited available hard drive space, we decided to operate it in a 10 frames per second regime, which still provides us with $\sim 3 \mathrm{~Tb}$ of data per night. The quantum efficiency is about $55 \%$, with read-out noise as low as $1 e^{-}$. The pixel scale is about $15^{\prime \prime}$ per pixel, and the channel field of view is about 100 square degrees. The limiting magnitude of the channel will be about $B \sim 12.5^{\mathrm{m}}$ in $0.1 \mathrm{~s}$, in both differential and direct imaging.

MMT-Kazan will be installed at the Engelgardt ob-

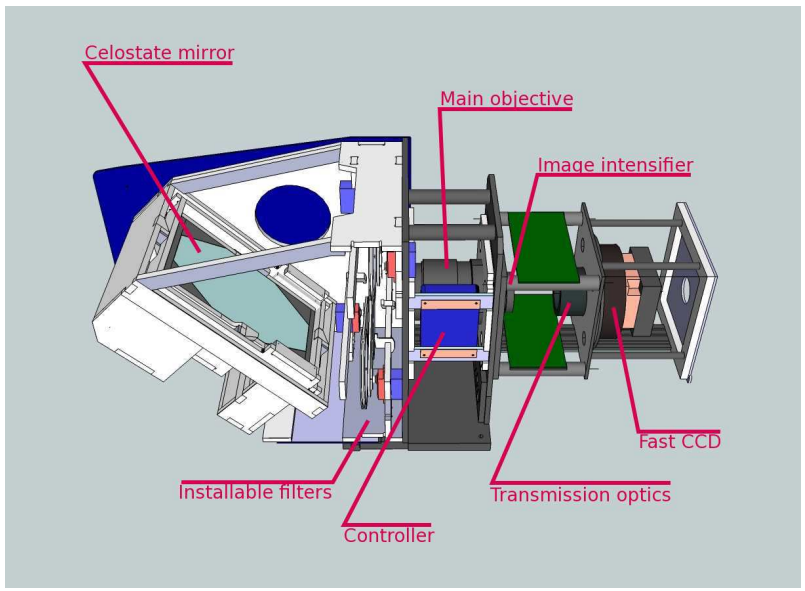

Figure 3. Scheme of the single channel mechanical design of the MMT-Spain variant.

servatory of Kazan Federal University, Kazan, Russia in summer 2013.

Both variants of MMT will use custom fork mounts based on a Skywatcher EQ6 head, each carrying two channels simultaneously. Each channel will be controlled by a dedicated Linux-based PC performing data acquisition (due to the absence of Linux drivers, for MMT-Spain we use one more Windows-based image grabber PC per channel, connected to data processing one via dedicated gigabit Ethernet cable), raw data storage, real-time data processing, as well as controlling the state of the filters and the celostate of the channel.

The operation of the complex as a whole is controlled by a central server which collects information on transients detected by channels and issues commands for repointing all of them towards it for a follow-up, choosing an appropriate combination of color and polarimetric filters, based on the brightness of the transient. It also checks the hardware state of channels, the weather conditions and the day/night status, starting and stopping the routine monitoring accordingly. 


\section{Strategy of Mini-MEgATORTORA OPERATION}

Mini-MegaTORTORA will perform routine observations of all the available sky in wide-field regime, which gives a $\sim 900$ square degrees field of view for MMTKazan. It will spend up to 20 minutes on each spot, selected to follow as much as possible the fields of view of space-borne gamma-ray telescopes according to information from the GCN network [2], while avoiding regions currently being observed by other monitoring systems such as "Pi of the Sky" [19], the regions close to the Moon or the horizon, and regions recently observed by the complex itself. In 8 hours of typical dark night, it will cover up to $\sim 20000$ square degrees, nearly half of the whole sky, and will return to each spot in about one day.

On each spot, each channel will collect about 10000 frames, which will allow scientists to study its variability on different time scales with different limits by co-adding consecutive frames (this co-addition will not be subject to coordinate re-binning and varying spatial sensitivity problems, as all frames are collected consecutively on the same detector imaging the same sky region with sufficiently good telescope tracking) co-adding of 100 frames may improve the limit by up to $2.5^{\mathrm{m}}$, while co-adding of 10000 frames may improve the limit by up to $5^{\mathrm{m}}$, depending on the temporal stability of the detector and the sky conditions, and also the quality of the flatfielding and dark frames. Frames co-added by 100 will be stored forever to form a timedomain atlas of the sky for further study, along with a time-domain photometric catalogue formed by measurements by means of fast aperture photometry (on a 100 frames / $10 \mathrm{~s}$ time scale, down to $B \sim 15^{\mathrm{m}}$ for MMT-Kazan) or slower PSF-fitting photometry (on a 10000 frames $/ 1000 \mathrm{~s}$ time scale, down to $B \sim 17.5^{\mathrm{m}}$ for MMT-Kazan). This catalogue will allow to study the variability of various classes of objects on time scales from 10 seconds to years, and also to detect slowly moving objects.

Compared with existing data from the ASAS-3 22] and NSVS [29] surveys, which have similar detection limits, we may expect up to $15-20$ millions of objects to be covered, with $\sim 100000$ being variable, and probably new classes of variable objects to be discovered due to better temporal resolution and cadence.

Real-time data processing, based on fast differential imaging and interlinking of events on several consecutive frames [4, 18], will allow us to detect both fast flashes (with durations longer than $0.3 \mathrm{~s}$ ) and rapidly moving satellites (with velocities up to half degree per second), as well as meteors (even meteors appearing on a single frame, as they are selected on the basis of their elongated shape), and roughly classify them on the fly. For transients, the light curve and coordinates will be stored, while for satellites - the trajectories will also be stored for further processing by more sophisticated methods in day time. If the transient is bright enough, and is not coincident with a known satellite or a bright star, the complex may be reconfigured to follow it up, pointing all the channels towards it and installing some combination of color and polarimetric filters to acquire both photometric and polarimetric information.

If all 9 channels are equipped with the same color filter, frame co-addition may yield up to $1^{\mathrm{m}}$ to the complex sensitivity, while in three-color mode it may yield up to $0.6^{\mathrm{m}}$. In polarimetric mode, the limit is nearly the same as in single-channel regime due to the light losses on polarimetric filters. The expected accuracy of polarimetry is about $10 \%$ at $10^{\mathrm{m}}$ and about $1 \%$ at $5^{\mathrm{m}}$.

\section{ACKNOWLEDGEMENTS}

This work was supported by Bologna University Progetti Pluriennali 2003, by grants of CRDF (No. RP1-2394-MO02), RFBR (No. 04-02-17555, 06-02-08313, 09-02-12053 and 12-02-00743-a), INTAS (04-78-7366), by the Presidium of the Russian Academy of Sciences Program, by a grant of the President of the Russian Federation in support of young Russian scientists and by a European Union grant (FP7 grant number 283783, GLORIA project). The construction of MMT-Kazan is being financed by Kazan Federal University. S.K. has also been supported by a grant from the Dynasty foundation. G.B. thanks Landau Network-Cenro Volta and the Cariplo Foundation for a fellowship and the Brera Observatory for hospitality. We thank Emilio Molinari, Stefano Covino and Cristiano Guidorzi for technical help in organizing TORTORA observations and for discussions of the results.

\section{REFERENCES}

[1] V. L. Afanasiev, V. V. Kalenichenko, I. D. Karachentsev. Detection of an intergalactic meteor particle with the 6-m telescope. Astrophysical Bulletin 62:301-310, 2007.

[2] S. D. Barthelmy. Observing strategies using GCN. American Institute of Physics Conference Series 428:129-133, 1998.

[3] G. Beskin, V. Bad'in, A. Biryukov, et al. FAVOR (FAst Variability Optical Registration) - A two-telescope complex for detection and investigation of short optical transients. Nuovo Cimento C 28:751-754, 2005.

[4] G. Beskin, A. Biryukov, S. Bondar, et al. Software for detection of optical transients in observations with rapid wide-field camera. Astronomische Nachrichten 325(6):676-676, 2004

[5] G. Beskin, S. Bondar, S. Karpov, et al. From TORTORA to MegaTORTORA - Results and Prospects of Search for Fast Optical Transients. Advances in Astronomy 2010:171569, 2010.

[6] G. Beskin, V. de-Bur, S. Karpov, et al. Search for optical signals from extra-terrestial intelligence at SAO RAS: past, present and futurte. Bulletin of Special Astrophysical Obervatory 60-61:217-225, 2007.

[7] G. Beskin, S. Karpov, S. Bondar, et al. TORTORA discovery of Naked-Eye Burst fast optical variability. American Institute of Physics Conference Series 1065:251-254, 2008. 
[8] G. Beskin, S. Karpov, S. Bondar, et al. Fast Optical Variability of a Naked-eye Burst - Manifestation of the Periodic Activity of an Internal Engine. ApJ 719:L10-L14, 2010.

[9] G. M. Beskin, V. Plokhotnichenko, C. Bartolini, et al. Catching the light curve of flaring GRBs: The opportunity offered by scanning telescopes. A\&AS 138:589-590, 1999.

[10] H. Bondi. Astronomy of the Future. QJRAstronSoc 11:443, 1970.

[11] K. N. Borozdin, S. P. Brumby, M. C. Galassi, et al. Real-Time Detection of Optical Transients with RAPTOR. Proceedings of the SPIE 4847:344-353, 2002.

[12] A. Burd, M. Cwiok, H. Czyrkowski, et al. Pi of the Sky all-sky, real-time search for fast optical transients. New Astronomy 10:409-416, 2005.

[13] A. J. Castro-Tirado, J. Soldán, M. Bernas, et al. The Burst Observer and Optical Transient Exploring System (BOOTES). AEAS 138:583-585, 1999.

[14] M. Cwiok, W. Dominik, G. Kasprowicz, et al. GRB 080319B prompt optical observation by Pi-of-the-Sky. GRB Coordinates Network Circular 7439:1, 2008.

[15] S. Karpov, D. Bad'in, G. Beskin, et al. FAVOR (FAst Variability Optical Registration) - two-telescope complex for detection and investigation of short optical transients. Astronomische Nachrichten 325:677-677, 2004.

[16] S. Karpov, G. Beskin, A. Biryukov, et al. Optical camera with high temporal resolution to search for transients in the wide field. Nuovo Cimento $C$ 28:747-750, 2005.

[17] S. Karpov, G. Beskin, S. Bondar, et al. Grb 080319b: Tortora synchronous observation. GRB Coordinates Network Circular 7452:1, 2008.

[18] S. Karpov, G. Beskin, S. Bondar, et al. Wide and Fast: Monitoring the Sky in Subsecond Domain with the FAVOR and TORTORA Cameras. Advances in Astronomy 2010:784141, 2010.

[19] S. Karpov, M. Sokolowski, E. Gorbovskoy. All Sky Coordination Initiative - simple service for wide-field monitoring systems to cooperate in searching for fast optical transients. Astronomical Society of India Conference Series p. in press, 2012.
[20] S. McBreen, F. Quilligan, B. McBreen, et al. Temporal properties of the short gamma-ray bursts. A 6 A 380:L31-L34, 2001.

[21] E. Molinari, S. Bondar, S. Karpov, et al. TORTOREM: two-telescope complex for detection and investigation of optical transients. Nuovo Cimento B 121:1525-1526, 2006.

[22] G. Pojmanski. The All Sky Automated Survey. Catalog of Variable Stars. I. 0 h - 6 hQuarter of the Southern Hemisphere. Acta Astronomica 52:397-427, 2002.

[23] J. L. Racusin, N. Gehrels, S. T. Holland, et al. GRB 080319B: Swift detection of an intense burst with a bright optical counterpart. GRB Coordinates Network Circular 7427:1, 2008.

[24] B. Schaefer. Celestial Optical Flash Rate Predictions and Observations. AJ 11:1363-1369, 1985.

[25] B. Schaefer. Optical Flash Background Rates. A\&A 174:338-343, 1987.

[26] V. F. Shvartsman, G. M. Beskin, R. E. Gershberg, et al. Minimum Rise Times in Uv-Ceti Type Flares. Soviet Astronomy Letters 14:97, 1988.

[27] T. Tamagawa, F. Usui, Y. Urata, et al. The search for optical emission on and before the GRB trigger with the WIDGET telescope. Nuovo Cimento C 28:771-774, 2005.

[28] P. Wozniak, W.T. Vestrand, J. Wren, H. Davis. GRB 080319B: RAPTOR observations of a naked eye burst. GRB Coordinates Network Circular 7464:1, 2008.

[29] P. R. Woźniak, W. T. Vestrand, C. W. Akerlof, et al. Northern Sky Variability Survey: Public Data Release. AJ 127:2436-2449, 2004.

[30] I. Zolotukhin, G. Beskin, A. Biryukov, et al. Optical camera with high temporal resolution to search for transients in the wide field. Astronomische Nachrichten 325:675-675, 2004. 